\title{
MicroRNA-889 plays a suppressive role in cell proliferation and invasion by directly targeting TAB1 in non-small cell lung cancer
}

\author{
ZHENG DONG ${ }^{1}$, BINGCHUN $\mathrm{LI}^{2}$ and XIAOJUAN WANG ${ }^{2}$ \\ Departments of ${ }^{1}$ Outpatients and ${ }^{2}$ Thoracic Surgery, Weifang People's Hospital, Weifang, Shandong 261041, P.R. China
}

Received November 5, 2018; Accepted April 9, 2019

DOI: $10.3892 / \mathrm{mmr} .2019 .10245$

\begin{abstract}
MicroRNAs (miRNAs) are frequently reported to be aberrantly expressed in non-small cell lung cancer (NSCLC) and are closely associated with aggressive tumor phenotypes. Hence, identification of cancer-related miRNAs in NSCLC may be helpful for improving the cure rate of NSCLC treatments. miR-889 has been demonstrated to be a novel cancer-associated miRNA that is aberrantly expressed and plays an important role in esophageal squamous cell carcinoma and hepatocellular carcinoma. However, the exact functions and precise molecular mechanisms through which miR-889 affects NSCLC progression are still unknown. In the present study, we report for the first time that miR-889 expression is low in NSCLC tissues and cell lines. Clinically, low miR-889 expression was found to be correlated with the TNM stage and distant metastasis in NSCLC patients. Functionally, miR-889 overexpression suppressed the proliferation and invasiveness of NSCLC cells in vitro and decreased NSCLC xenograft tumor growth in mice. Furthermore, TGF- $\beta$-activated kinase 1-binding protein 1 (TAB1) was confirmed as a direct target gene of miR-889 in NSCLC cells. TAB1 was revealed to be overexpressed in NSCLC tissue samples and was inversely correlated with miR-889 levels. Moreover, a TAB1 knockdown had effects similar to that of miR-889 overexpression, whereas restoration of TAB1 expression counteracted the actions of miR-889 in NSCLC cells. Overall, the present results indicated that miR-889 inhibits the aggressive behaviors of NSCLC by directly targeting TAB1 mRNA, thus highlighting the importance of the miR-889/TAB1 pathway in the malignant progression of NSCLC.
\end{abstract}

\section{Introduction}

Lung cancer, a highly lethal malignant tumor, is a common cancer and represents a leading cause of cancer-related

Correspondence to: Professor Xiaojuan Wang, Department of Thoracic Surgery, Weifang People's Hospital, 151 Guangwen Road, Weifang, Shandong 261041, P.R. China

E-mail: wang_xjwf@163.com

Key words: microRNA-889, non-small cell lung cancer, proliferation, invasion, TGF- $\beta$-activated kinase 1-binding protein 1 deaths worldwide (1). Approximately $85 \%$ of lung cancer cases are classified histopathologically as non-small cell lung cancer (NSCLC), which includes lung adenocarcinoma, lung squamous carcinoma, and large cell lung cancer (2). Notable improvements in the diagnosis and treatment of NSCLC have been achieved in the past decades; unfortunately, the prognosis of patients with NSCLC remains unfavorable, with a 5-year overall survival rate of only $11 \%$ (3). Tumor recurrence and metastasis are primarily responsible for the unsatisfying clinical outcomes for NSCLC patients $(4,5)$. Multiple risk factors and signaling pathways have been implicated in the pathogenesis of NSCLC; however, the precise molecular mechanisms are yet to be fully clarified (6). Therefore, elucidation of the molecular mechanisms underlying the initiation and progression of NSCLC is urgently necessary and may help to identify novel diagnostic biomarkers and therapeutic methods to improve the prognosis of NSCLC patients.

MicroRNAs (miRNAs) are a family of noncoding, single-stranded, short RNA molecules that have been demonstrated to serve as an endogenous means of RNA interference (7). miRNAs can directly recognize and interact with a partially complementary recognition sequence in the 3' untranslated region (3'-UTRs) of the mRNA of a target gene and cause degradation of the mRNA and/or translation suppression (8). It has been well documented that miRNAs are implicated in a variety of physiological and pathological processes, including the initiation and progression of human cancers $(9,10)$. A wide range of miRNAs are differentially expressed in NSCLCs (11-13). For example, miR-217 expression is often low in NSCLC. NSCLC patients with low miR-217 expression exhibit shorter overall survival than do the patients with high miR-217 expression (14). These aberrantly expressed miRNAs may perform oncogenic or tumor-suppressive functions and contribute to the oncogenesis and progression of NSCLC by regulating multiple crucial biological processes $(15,16)$. These findings indicate that an in-depth investigation into the participation and mechanisms of action of cancer-related miRNAs in NSCLC may facilitate the search for novel therapeutic targets in NSCLC.

Certain studies have identified miR-889 as a novel cancer-associated miRNA that is aberrantly expressed and plays an important role in esophageal squamous cell carcinoma (17) and hepatocellular carcinoma (18). Nonetheless, the exact functions and precise molecular mechanisms via which miR-889 affects NSCLC progression are still unclear. Thus, 
in the present study, the quantification of miR-889 expression was attempted and the potential involvement and mechanism of action of miR-889 in NSCLC were investigated. The present findings are expected to provide novel insights into the pathogenesis of NSCLC and/or a promising target for the treatment of patients with this disease.

\section{Materials and methods}

Patients and tissue specimens. The procedures of our present study were approved by the Ethics Committee of Weifang People's Hospital (Weifang, China) and written informed consent was also provided by all patients. Samples of primary NSCLC tissues and adjacent normal tissues were collected from 53 patients (30 males, 23 females; age range, 47-71 years) who had received surgical resection at Weifang People's Hospital between June 2016 and August 2017. None of these patients had been treated preoperatively with either radiotherapy or chemotherapy. All tissues were immediately snap-frozen in liquid nitrogen and then stored at $-80^{\circ} \mathrm{C}$ for further RNA extraction.

Cell culture. A non-tumorigenic bronchial epithelium cell line, BEAS-2B, and four human NSCLC cell lines (A549, SK-MES-1, H522, and H460) were purchased from the Shanghai Institute of Biochemistry and Cell Biology (Shanghai, China). All cells were routinely cultured in Dulbecco's modified Eagle's medium (DMEM) containing 10\% fetal bovine serum (FBS; both from Gibco; Thermo Fisher Scientific, Inc., Waltham, MA, USA) and $1 \%$ penicillin/streptomycin mixture (Sigma-Aldrich; Merck KGaA, Darmstadt, Germany). The cultures were maintained at $37^{\circ} \mathrm{C}$ in a humidified atmosphere containing $5 \% \mathrm{CO}_{2}$.

miRNA mimics, small interfering RNA (siRNA) and plasmid transfection. miR-889 mimics and corresponding miRNA mimics negative control (miR-NC), siRNA targeting the expression of TAB1 (si-TAB1) and negative control siRNA (si-NC) were constructed by GenePharma (Shanghai, China). The si-TAB1 sequence was 5'-GGAUGAGCUCUUCCGUCU UTT-3' and the si-NC sequence was 5'-UUCUCCGAACGU GUCACGUTT-3'. TAB1 expression vector pcDNA3.1-TAB1 (pcTAB1) and empty vector (pcDNA3.1) was obtained from Guangzhou RiboBio Co., Ltd. (Guangzhou, China). Before transfection, cells in logarithmic phase were plated into 6-well plates with a density of $4 \times 10^{5}$ cells/well. When the culture confluency reached $60-70 \%$, the cells were transfected with the miR-889 mimics (100 pmol), miR-NC (100 pmol), si-TAB1 (100 pmol), si-NC (100 pmol), pcTAB1 $(4 \mu \mathrm{g})$ or pcDNA3 $(4 \mu \mathrm{g})$ using Lipofectamine ${ }^{\mathrm{TM}} 2000$ (Invitrogen; Thermo Fisher Scientific, Inc.). All of the transfection procedures were based on the product specifications. Reverse transcription-quantitative polymerase chain reaction (RT-qPCR) and Transwell Matrigel invasion assays were performed $48 \mathrm{~h}$ after transfection. The MTT assay and western blotting were performed at 24 and $72 \mathrm{~h}$ post-transfection, respectively.

RNA extraction and RT-qPCR analysis. Total RNA was extracted from tissue samples or cultured cell lines using a TRIzol ${ }^{\circledR}$ reagent (Invitrogen; Thermo Fisher Scientific, Inc.).
All-in-One ${ }^{\mathrm{TM}}$ miRNA qRT-PCR Detection kit (GeneCopoeia, Inc., Rockville, MD, USA) was used to detect miR-889 expression. The thermocycling conditions were as follows: $95^{\circ} \mathrm{C}$ for $10 \mathrm{~min}$, and 45 cycles of denaturation at $95^{\circ} \mathrm{C}$ for $15 \mathrm{sec}$ and annealing/elongation at $60^{\circ} \mathrm{C}$ for $15 \mathrm{sec}$. To quantify TAB1 mRNA expression, total RNA was reverse-transcribed into cDNA using a PrimeScript RT reagent kit (Takara Biotechnology Co., Ltd., Dalian, China). The temperature protocol for TAB1 mRNA reverse transcription was as follows: $37^{\circ} \mathrm{C}$ for $15 \mathrm{~min}$ and $85^{\circ} \mathrm{C}$ for 5 second. The quantitative PCR was carried out using an ABI 7500 Sequence Detection system (Applied Biosystems; Thermo Fisher Scientific, Inc.) and a SYBR Premix Ex Taq ${ }^{\mathrm{TM}}$ (Takara Biotechnology Co., Ltd). The thermocycling conditions for TAB1 mRNA qPCR was as follows: $5 \mathrm{~min}$ at $95^{\circ} \mathrm{C}$, followed by 40 cycles of $95^{\circ} \mathrm{C}$ for $30 \mathrm{sec}$ and $65^{\circ} \mathrm{C}$ for $45 \mathrm{sec}$. U6 small nuclear RNA and GAPDH were used as the internal controls for miR-889 and TAB1 mRNA, respectively. All reactions were run in triplicate and relative gene expression was analyzed using the $2^{-\Delta \Delta \mathrm{Cq}}$ method (19). The primers were designed as follows: miR-889 forward, 5'-ACACTCCAGCTGGGTTAATATCGGACAAC-3', and reverse, 5'-TGGTGTCGTGGAGTCG-3'; U6 forward, 5'-GCT TCGGCAGCACATATACTAAAAT-3', and reverse, 5'-CGC TTCACGAATTTGCGTGTCAT-3'; TAB1 forward, 5'-ATG AGCTCTTCCGTCTTTCG-3', and reverse, 5'-ATCCCCACC TGCTTGATCT-3'; and GAPDH forward, 5'-GGAGCGAGA TCCCTCCAAAAT-3' and reverse, 5'-GGCTGTTGTCAT ACTTCTCATGG-3'.

MTT assay. An MTT assay was performed to determine cellular proliferation. In brief, transfected cells were inoculated in 96-well plates with a density of 3,000 cells/well. Then the cells were incubated at $37^{\circ} \mathrm{C}$ supplied with $5 \% \mathrm{CO}_{2}$. for 0-3 days. The MTT assay was carried out every day by adding $20 \mu \mathrm{l}$ of MTT reagent $(5 \mathrm{mg} / \mathrm{ml}$; Beyotime Institute of Biotechnology, Haimen, China) into each well. Following incubation at $37^{\circ} \mathrm{C}$ for an additional $4 \mathrm{~h}$, the culture medium containing MTT solution was replaced with $150 \mu$ l of dimethyl sulfoxide (DMSO). A total of $15 \mathrm{~min}$ after incubation, the absorbance value of each well was detected at a $450 \mathrm{~nm}$ wavelength using an enzyme-linked immunosorbent assay reader (Bio-Rad Laboratories, Inc., Hercules, CA, USA).

Transwell Matrigel invasion assay. Transwell insert chambers ( $8 \mu \mathrm{m}$ pore size) covered with Matrigel (both from BD Biosciences, San Jose, CA, USA) were utilized to evaluate the invasive ability of NSCLC cells. Transfected cells were collected and suspended in FBS-free culture medium. A total of $5 \times 10^{4}$ cells were added into the upper chambers. In the lower chambers, $500 \mu \mathrm{l}$ DMEM containing 20\% FBS was added to serve as a chemoattractant. Subsequent to incubation at $37^{\circ} \mathrm{C}$ with $5 \% \mathrm{CO}_{2}$ for $24 \mathrm{~h}$, the non-invasive cells were gently wiped away with a cotton swab. The invasive cells that invaded through the pores were fixed with $100 \%$ methanol at room temperature for $30 \mathrm{~min}$, stained with $0.5 \%$ crystal violet at room temperature for $30 \mathrm{~min}$, and washed with phosphate buffer solution. Images of five different fields were captured from each insert, and the number of invasive cells was counted under an inverted light microscope (x200, magnification; Olympus Corporation, Tokyo, Japan). 
Xenograft assay. A total of eight 6-week-old BALB/c male nude mice $(20 \mathrm{~g})$ were purchased from the Shanghai Laboratory Animal Center (Chinese Academy of Sciences, Shanghai, China). All animals were maintained under specific pathogen-free conditions at $25^{\circ} \mathrm{C}, 50 \%$ relative humidity, a $10 \mathrm{~h}$ light/14 h dark cycle and had ad libitum access to food and water. The animals were divided into two groups $(n=4$ mice/group), one injected with miR-NC-transfected cells and the other injected with miR-889 mimic-transfected cells. The width and length of the xenograft formed was detected with a vernier caliper. Tumor volume was calculated by the following formula: Volume $\left(\mathrm{mm}^{3}\right)=$ width $^{2}\left(\mathrm{~mm}^{2}\right) \times$ length $(\mathrm{mm}) / 2$. At the end of the assay, all nude mice were sacrificed and the excited xenograft was weighted. All animal experiments were carried out in accordance with the Guide for Care and Use of Laboratory Animal and all experimental protocols were approved by the Animal Ethics Committee of the Weifang People's Hospital.

Target prediction. Three online miRNA target prediction software, including miRDB (http://www.mirdb.org/), miRanda (http://www.microrna.org), and TargetScan (http://www. targetscan.org/), were used to predict the potential target genes of miR-889.

Luciferase reporter assay. The 3'-UTR region of TAB1 containing the predicted wild-type and mutant miR-889 binding sites was amplified by GenePharma and individually cloned into the pmiR-RB-REPORT ${ }^{\mathrm{TM}}$ vector (Promega Corporation, Madison, WI, USA). For the reporter assays, cells were plated into 24 -well plates, and co-transfected with wild type or mutant reporter plasmid and miR-889 mimics or miR-NC using Lipofectamine ${ }^{\mathrm{TM}}$ 2000, according to the manufacturer's instructions. Forty-eight hours later, the luciferase activity of transfected cells was determined using a Dual-Luciferase Reporter Assay System (Promega Corporation). Firefly luciferase activity was normalized against Renilla luciferase activity.

Western blot analysis. Total protein was isolated from tissues or cells using cell lysis buffer (Cell Signaling Technology, Danvers, MA, USA). The concentration of total protein was determined using a bicinchoninic acid protein assay (Pierce; Thermo Fisher Scientific, Inc.). Equal amounts of protein $(30 \mu \mathrm{g})$ were separated by $10 \%$ sodium dodecyl sulfate polyacrylamide gels electrophoresis and transferred onto polyvinylidene fluoride membranes (Beyotime Institute of Biotechnology), followed by blocking with 5\% dried skimmed milk in Tris-buffered saline and $0.1 \%$ Tween-20 (TBS-T) at room temperature for $1 \mathrm{~h}$. The membranes were subsequently incubated with the following primary antibodies at $4^{\circ} \mathrm{C}$ overnight: Rabbit anti-human TAB1 antibody (cat. no. ab76412; 1:1,000 dilution) and rabbit anti-human GAPDH antibody (cat. no. ab128915; 1:1,000 dilution; both from Abcam, Cambridge, UK). The membranes were then probed with horseradish peroxidase-conjugated secondary antibody (cat. no. ab205718; 1:5,000 dilution; Abcam,) for $2 \mathrm{~h}$ at room temperature. Finally, the protein bands were visualized using an enhanced chemiluminescence reagent (Bio-Rad Laboratories, Inc., Hercules, CA, USA). GAPDH was used as the internal control. Quantity
One software version 4.62 (Bio-Rad Laboratories, Inc.) was used for densitometry analysis.

Statistical analysis. All assays were repeated at least three times. All data are presented as the mean \pm standard deviation, and analyzed using SPSS 19.0 software (IBM Corp., Armonk, NY, USA). All functional experiments were repeated three times. Differences between two groups were examined using an unpaired Student's t-test, or a paired Student's t-test to compare expression data from NSCLC and adjacent normal tissues. One-way ANOVA with Tukey's post-hoc test was used for the comparison between multiple groups. A $\chi^{2}$ test was performed to evaluate the association between miR-889 and the clinicopathological characteristics of NSCLC patients. A correlation between miR-889 and TAB1 mRNA expression was determined through Spearman's correlation analysis. $\mathrm{P}<0.05$ was considered to indicate a statistically significant difference.

\section{Results}

miR-889 is downregulated in NSCLC tissue samples and cell lines. In total, 53 pairs of NSCLC tissue samples and adjacent normal tissue samples were collected. Total RNA was isolated from these tissues, and RT-qPCR was performed to assess miR-889 expression. The data revealed that the expression of miR-889 was notably lower in NSCLC tissue samples than in adjacent normal tissues $(\mathrm{P}<0.05$; Fig. 1A). In addition, miR-889 expression in four human NSCLC cell lines (A549, SK-MES-1, H522, and H460) was determined by RT-qPCR. When compared with non-tumorigenic bronchial epithelium BEAS-2B cells, miR-889 expression was lower in all four NSCLC cell lines to varying degrees $(\mathrm{P}<0.05$; Fig. 1B). These data indicated that miR-889 was underexpressed in both NSCLC tissue samples and cell lines.

Downregulated miR-889 is correlated with the clinical characteristics of patients with NSCLC. To clarify the possible clinical meaning of low miR-889 expression in NSCLC, all enrolled NSCLC patients were categorized into two subgroups, 'low miR-889 expression' and 'high miR-889 expression', based on the median value of miR-889 expression in NSCLC tissue samples. Statistical analysis revealed that underexpression of miR-889 was significantly correlated with TNM stage $(\mathrm{P}=0.019)$ and distant metastasis $(\mathrm{P}=0.020)$; however, no significant associations with other pathological characteristics were observed, including sex $(\mathrm{P}=0.477)$, age $(\mathrm{P}=0.685)$, tumor size $(\mathrm{P}=0.407)$, histological tumor type $(\mathrm{P}=0.449)$, or tumor differentiation status $(\mathrm{P}=0.317$; Table $\mathrm{I})$. These results indicated that underexpression of miR- 889 may be implicated in the pathogenesis of NSCLC.

miR-889 overexpression inhibits the proliferation and invasiveness of NSCLC cells. To assess whether miR-889 affects NSCLC progression, cell lines H522 and H460 (possessing relatively lower miR-889 expression among the four assessed NSCLC cell lines) were selected to conduct the following experiments. miR-889 in H522 and H460 cells was overexpressed by transfection with miR-889 mimics ( $\mathrm{P}<0.05$; Fig. $2 \mathrm{~A}$ ). Then, an MTT assay was carried out to quantify cell proliferation; it was revealed that miR-889 upregulation significantly 

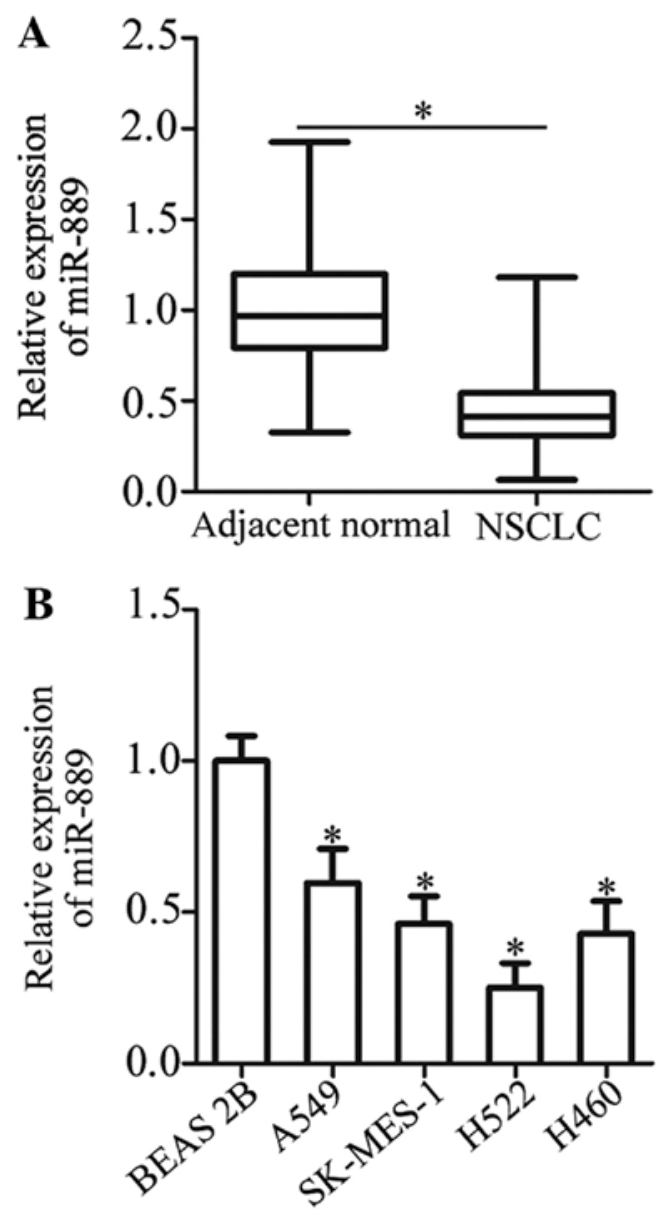

Figure 1. miR-889 is downregulated in NSCLC tissues and cell lines. (A) The expression status of miR- 889 was determined by RT-qPCR in 53 pairs of NSCLC tissues and adjacent normal tissues. "P $<0.05$ vs. adjacent normal tissues. (B) The expression level of miR-889 in four human NSCLC cell lines (A549, SK-MES-1, H522, and H460) and a non-tumorigenic bronchial epithelium BEAS-2B cell line was detected by RT-qPCR. ${ }^{*} \mathrm{P}<0.05$ vs. BEAS-2B. NSCLC, non-small cell lung cancer.

inhibited H522 and H460 cell proliferation when compared with the miR-NC group $(\mathrm{P}<0.05$; Fig. 2B). Furthermore, a Transwell Matrigel invasion assay was employed to investigate the regulation of cell invasion by miR-889 in NSCLC. Ectopic miR-889 expression decreased the capacity for invasion in $\mathrm{H} 522$ and $\mathrm{H} 460$ cells relative to the cells treated with miR-NC $(\mathrm{P}<0.05$; Fig. 2C). Collectively, these findings indicated that miR-889 may perform tumor-suppressive functions in NSCLC carcinogenesis.

TAB1 is a direct target gene of miR-889 in NSCLC cells. To illustrate the potential molecular mechanisms by which miR- 889 exerts its influence on NSCLC progression, bioinformatics tools, including miRDB, miRanda, and TargetScan, were applied to find a potential target gene (i.e., target mRNA) of miR-889. This analysis indicated a possible binding site of miR- 889 in the 3'-UTR of TAB1 (Fig. 3A). TAB1 was selected for further analyses since this gene may participate in the initiation and progression of NSCLC (20). The luciferase reporter assay was conducted to determine whether miR-889 targets the 3'-UTR of TAB1 mRNA directly. To this end, luciferase reporter vectors were chemically synthesized and co-transfected into H522 and
Table I. Correlation of miR-889 expression and clinicopathological characteristics of patients with non-small cell lung cancer.

\begin{tabular}{|c|c|c|c|}
\hline \multirow[b]{2}{*}{ Characteristics } & \multicolumn{2}{|c|}{$\operatorname{miR}-889$} & \multirow[b]{2}{*}{ P-value } \\
\hline & $\begin{array}{c}\text { Low } \\
\text { expression }\end{array}$ & $\begin{array}{c}\text { High } \\
\text { expression }\end{array}$ & \\
\hline Sex & & & 0.477 \\
\hline Male & 14 & 16 & \\
\hline Female & 13 & 10 & \\
\hline Age (years) & & & 0.685 \\
\hline$<65$ & 12 & 13 & \\
\hline$\geq 65$ & 15 & 13 & \\
\hline Tumor size $(\mathrm{cm})$ & & & 0.407 \\
\hline$<5$ & 18 & 20 & \\
\hline$\geq 5$ & 9 & 6 & \\
\hline Histological tumor type & & & 0.449 \\
\hline Adenocarcinoma & 16 & 18 & \\
\hline Squamous cell carcinoma & 11 & 8 & \\
\hline Tumor differentiation & & & 0.317 \\
\hline I-II & 6 & 9 & \\
\hline III-IV & 21 & 17 & \\
\hline TNM stage & & & $0.019^{\mathrm{a}}$ \\
\hline I-II & 7 & 15 & \\
\hline III+IV & 20 & 11 & \\
\hline Distant metastasis & & & $0.020^{\mathrm{a}}$ \\
\hline Negative & 9 & 17 & \\
\hline Positive & 18 & 9 & \\
\hline
\end{tabular}

a $<<0.05$. NSCLC, non-small cell lung cancer.

H460 cells along with miR-889 mimics or miR-NC. The exogenous miR-889 expression reduced the luciferase activity of the vector that carried the wild-type 3 '-UTR of TAB1 $(\mathrm{P}<0.05)$. By contrast, mutation of the miR-889 binding site in the 3'-UTR of TAB1 abrogated the inhibitory effect of miR- 889 overexpression on the luciferase activity (Fig. 3B).

To confirm that TAB1 contributes to NSCLC pathogenesis, RT-qPCR analysis was carried out to assess TAB1 mRNA expression in NSCLC tissue samples. The data indicated that the mRNA expression of TAB1 was notably higher in NSCLC tissue samples than in the adjacent normal tissues $(\mathrm{P}<0.05$; Fig. 3C). Furthermore, miR-889 expression was revealed to be negatively correlated with TAB1 mRNA levels among NSCLC tissue samples ( $\mathrm{r}=-0.5077, \mathrm{P}=0.0001$; Fig. 3D). Moreover, TAB1 mRNA and protein levels in H522 and H460 cells transfected with miR-889 mimics or miR-NC were examined by RT-qPCR and western blot analysis, respectively. Transfection of the miR-889 mimics significantly decreased TAB1 expression in $\mathrm{H} 522$ and $\mathrm{H} 460$ cells at both the mRNA $(\mathrm{P}<0.05$; Fig. 3E) and protein levels $(\mathrm{P}<0.05$; Fig. 3F). Thus, it was demonstrated that TAB1 mRNA is a direct target of miR-889 in NSCLC cells. 

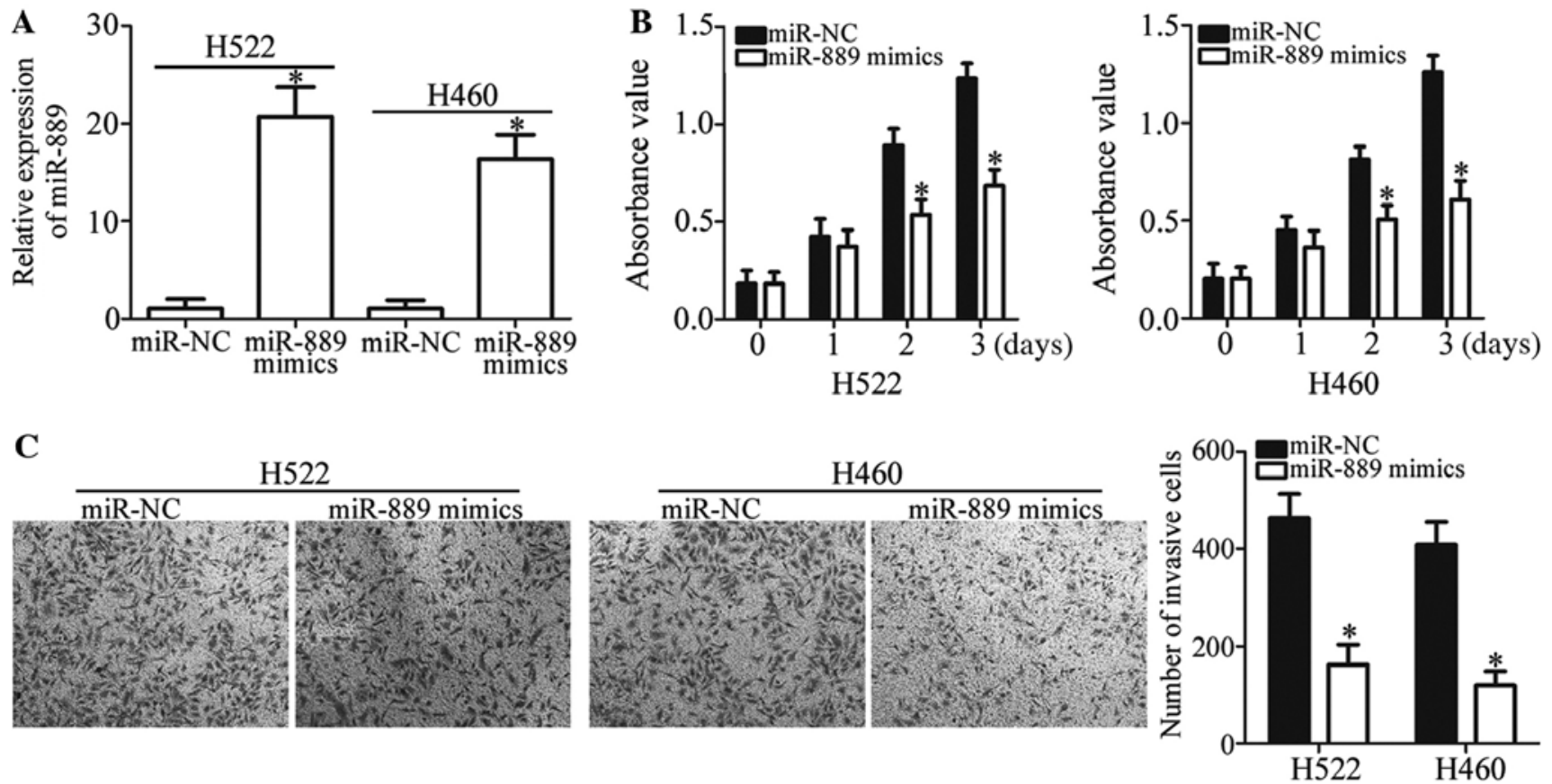

Figure 2. miR-889 attenuates the proliferation and invasion of NSCLC cells. (A) Quantitation of the miR-889 expression was performed in H522 and H460 cells after transfection of miR-889 mimics or miR-NC. "P<0.05 vs. miR-NC. (B) The cell proliferation was assessed by MTT assay in H522 and H460 cells that were treated with miR- 889 mimics or miR-NC. ${ }^{*}<<0.05$ vs. miR-NC. (C) Representative images and quantitation of the Transwell Matrigel invasion assay were carried out in miR-889 mimics- or miR-NC-transfected H522 and H460 cells (x200, magnification). ${ }^{*} \mathrm{P}<0.05$ vs. miR-NC. NSCLC, non-small cell lung cancer.

A
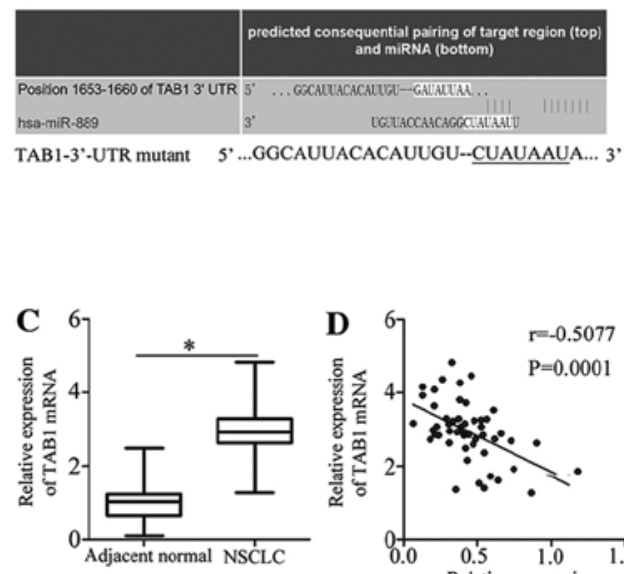

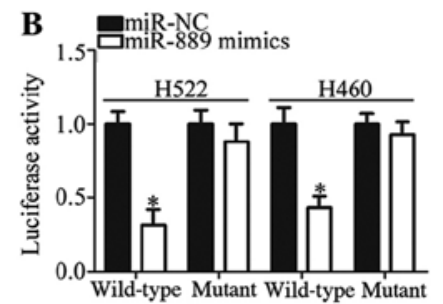

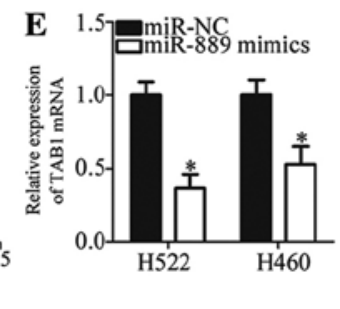

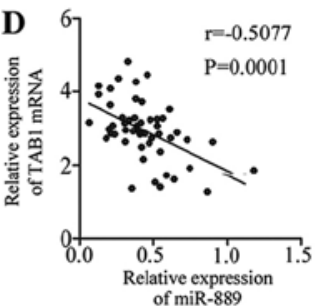

$\mathbf{F}$
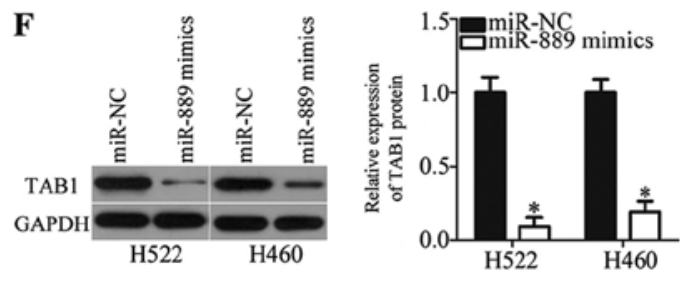

Figure 3. TAB1 is a direct target of miR-889 in NSCLC cells. (A) The predicted miR-889 wild-type binding site in the 3'-UTR of TAB1 using bioinformatics tools and the mutated 3'-UTR is presented. (B) H522 and H460 cells were co-transfected with a luciferase reporter vector that contained the wild type or mutant binding site of TAB1-3'-UTR plus miR- 889 mimics or miR-NC. Luciferase activity was detected $48 \mathrm{~h}$ after transfection. " $\mathrm{P}<0.05 \mathrm{vs}$. miR-NC. (C) The expression of TAB1 mRNA in NSCLC tissues was notably higher than that in adjacent normal tissues. " $\mathrm{P}<0.05$ vs. adjacent normal tissues. (D) miR-889 expression was inversely correlated with the expression of TAB1 mRNA in NSCLC tissues. $\mathrm{r}=-0.5077, \mathrm{P}=0.001$. (E) Relative TAB1 mRNA expression was detected by RT-qPCR in H522 and H460 cells after miR- 889 mimics or miR-NC transfection. ${ }^{*} \mathrm{P}<0.05$ vs. miR-NC. (F) The protein level of TAB1 in H522 and H460 cells following transfection with miR- 889 mimics or miR-NC was determined by western blot analysis. "P<0.05 vs. miR-NC. TAB1, TGF- $\beta$-activated kinase 1-binding protein 1; NSCLC, non-small cell lung cancer.

TAB1 silencing has effects similar to those of miR-889 mimics on the malignant phenotype of NSCLC cells in vitro. To precisely determine the involvement of TAB1 in NSCLC, endogenous TAB1 expression was knocked down in H522 and $\mathrm{H} 460$ cells by transfection with siRNA against TAB1 (si-TAB1). This transfection efficiently decreased TAB1 protein expression in $\mathrm{H} 522$ and $\mathrm{H} 460$ cells, as evidenced by western blot analysis $(\mathrm{P}<0.05$; Fig. $4 \mathrm{~A})$. Then, MTT and Transwell Matrigel invasion assays were performed to evaluate the changes in proliferative and invasive abilities of H522 and H460 cells after transfection with si-TAB1 or si-NC. The knockdown of TAB1 inhibited the proliferation $(\mathrm{P}<0.05$; Fig. 4B) and invasiveness $(\mathrm{P}<0.05$; Fig. $4 \mathrm{C})$ of H522 and H460 cells. These observations confirmed that TAB1 inhibition may imitate the tumor-suppressive roles of miR-889 mimics in NSCLC cells, further supporting the 

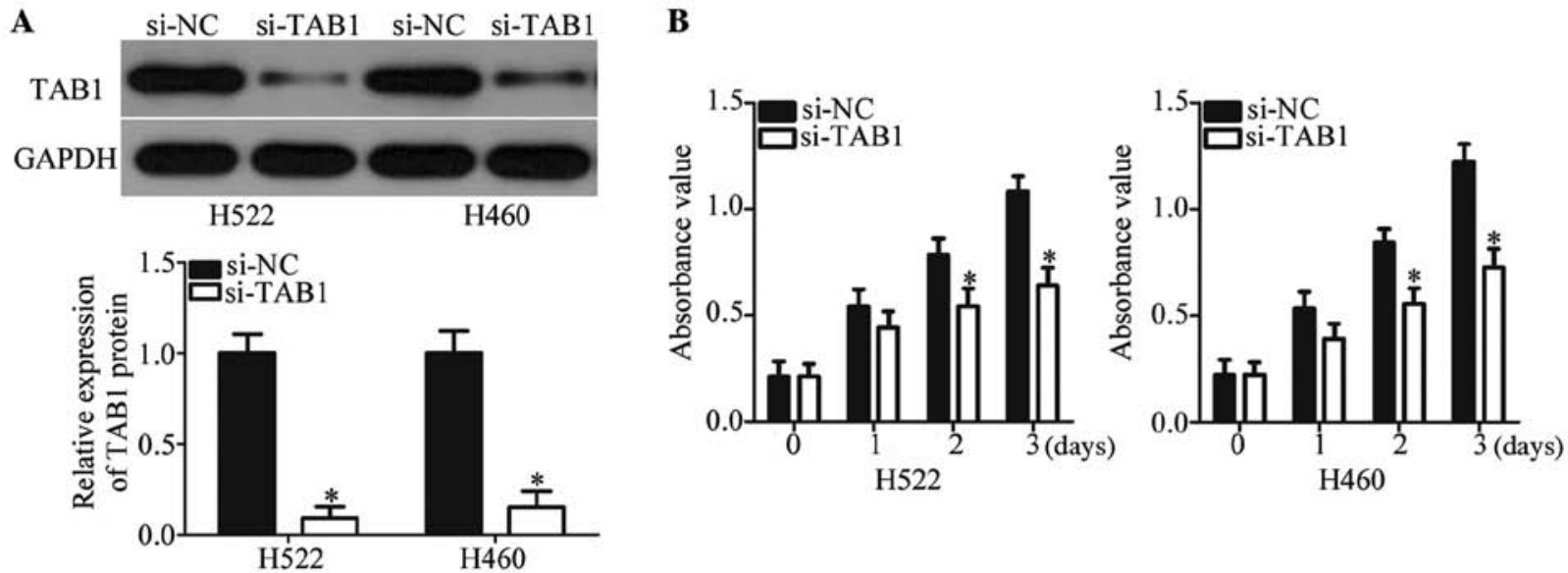

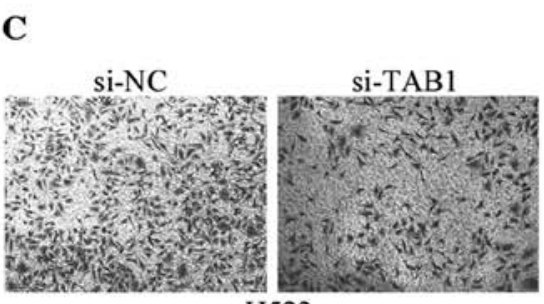

H522

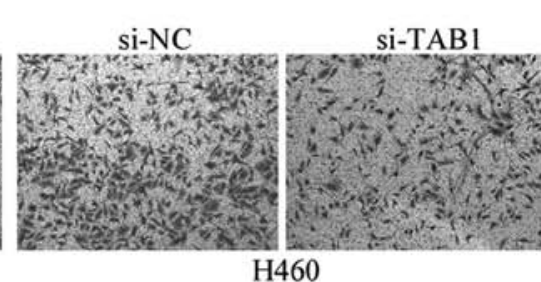

$\mathrm{H} 460$

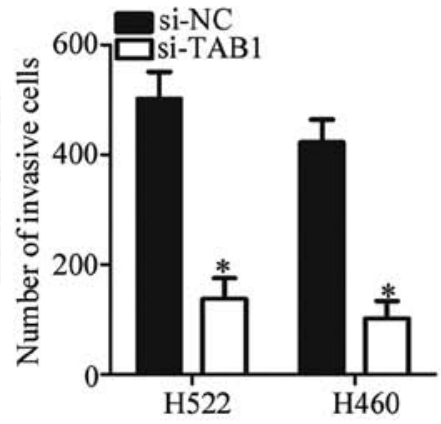

Figure 4. TAB1 inhibition suppresses cell proliferation and invasion in NSCLC. H522 and H460 cells were transfected with si-TAB1 or si-NC. (A) Following $72 \mathrm{~h}$ of incubation, western blot analysis was used to determine TAB1 proliferation. ${ }^{*} \mathrm{P}<0.05$ vs. si-NC. (B) An MTT assay was performed to evaluate the proliferation of indicated cells. "P<0.05 vs. si-NC. (C) The effect of TAB1 silencing in H522 and H460 cells was assessed using Transwell Matrigel invasion assay (x200, magnification). " $\mathrm{P}<0.05$ vs. si-NC. TAB1, TGF- $\beta$-activated kinase 1-binding protein 1 ; NSCLC, non-small cell lung cancer.

notion that TAB1 mRNA is a target of miR-889 in NSCLC cells.

TAB1 reintroduction counteracts the antitumor actions of miR-889 in NSCLC cells. To confirm that TAB1 is involved in the miR-889-induced tumor-suppressive effects in NSCLC cells, rescue experiments were conducted next by restoring TAB1 expression in miR-889 mimic-transfected H522 and H460 cells. Firstly, pcDNA3.1 or pc-TAB1 was introduced into H522 and H460 cells. RT-qPCR analysis confirmed that the expression level of TAB1 mRNA was increased in pc-TAB1-transfected $\mathrm{H} 522$ and $\mathrm{H} 460$ cells $(\mathrm{P}<0.05$; Fig. 5A). Western blotting indicated that co-transfection with TAB1 expression vector pcDNA3.1-TAB1 (pc-TAB1) notably recovered the TAB1 protein amount that was significantly decreased by miR-889 mimics $(\mathrm{P}<0.05$; Fig. 5B). Functional experiments revealed that the reintroduction of TAB1 reversed the anti-proliferative $(\mathrm{P}<0.05$; Fig. 5C) and anti-invasive ( $\mathrm{P}<0.05$; Fig. 5D) effects of miR-889 upregulation in H522 and H460 cells. Overall, these results indicated that miR-889 exerts its anticancer actions on NSCLC at least partly by directly suppressing TAB1 expression.

miR-889 inhibits NSCLC tumor growth in vivo. A xenograft experiment was next conducted to examine the influence of miR-889 overexpression on in vivo tumor growth of NSCLC cells. H460 cells transfected with miR-889 mimics or miR-NC were subcutaneously injected into the flanks of nude mice. The tumor volume was measured for 1 month. Upregulation of miR-889 significantly decreased the tumor growth in vivo $(\mathrm{P}<0.05$; Fig. $6 \mathrm{~A}$ and $\mathrm{B})$. At the end of the experiment, all the nude mice were euthanized, and the excised xenografts were weighed. The results indicated that the xenograft tumors derived from miR-889-overexpressing H460 cells had lower weight than did the xenograft tumors in the miR-NC group $(\mathrm{P}<0.05$; Fig. $6 \mathrm{C})$. Then, miR-889 expression was quantitated in the excised xenograft tumors and it was revealed that miR- 889 was still overexpressed in the xenograft tumors of the 'miR-889 mimics' group $(\mathrm{P}<0.05$; Fig. $6 \mathrm{D})$. Furthermore, the protein amount of TAB1 in the xenograft tumors was significantly decreased by miR-889 overexpression, as revealed by western blot analysis (Fig. 6E). These observations indicated that miR-889 upregulation inhibited the tumor growth of NSCLC cells in vivo by suppressing TAB1 expression.

\section{Discussion}

miRNAs are frequently reported to be aberrantly expressed in NSCLC and closely related with aggressive phenotypes (21). Accumulating evidence has validated miRNAs as potential biomarkers for the diagnosis and prognosis as well as therapeutic targets for patients with NSCLC $(15,22,23)$. Hence, identification of cancer-associated miRNAs in NSCLC may be helpful for improving the curative effects. In the present 

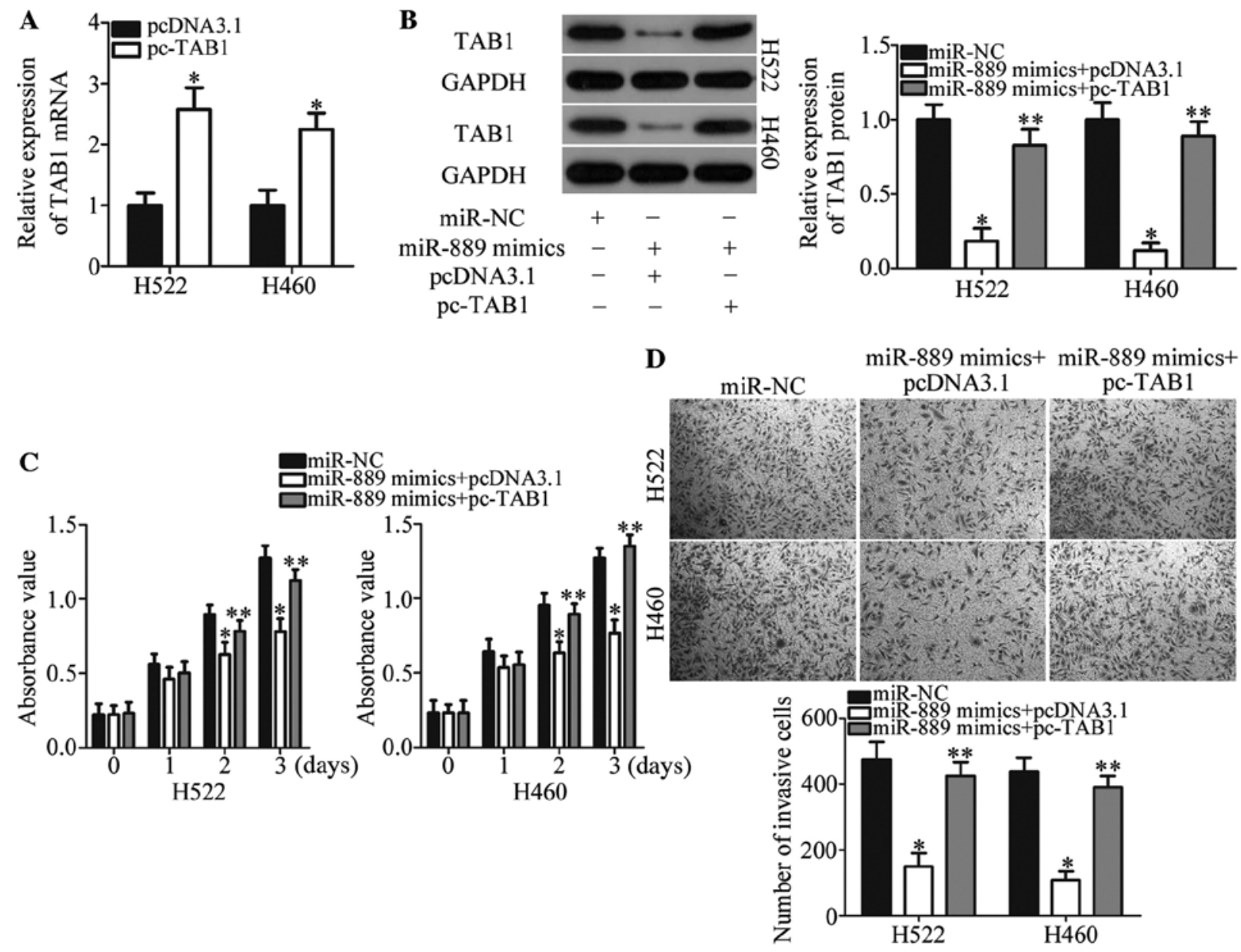

Figure 5. Recovered TAB1 expression reverses the antitumor effects of miR-889 in NSCLC cells. (A) RT-qPCR was utilized for the determination of TAB1 mRNA expression in $\mathrm{H} 522$ and $\mathrm{H} 460$ cells after pcDNA3.1 or pc-TAB1 transfection. ${ }^{*} \mathrm{P}<0.05$ vs. pcDNA3.1. (B) TAB1 protein expression in H522 and H460 cells co-transfected with miR-889 mimics and pc-TAB1 or pcDNA3.1 was demonstrated by western blot analysis. ${ }^{*} \mathrm{P}<0.05$ vs. miR-NC. ${ }^{* *} \mathrm{P}<0.05$ vs. miR-889 mimics+pcDNA3.1. (C and D) Cellular proliferation and invasion of aforementioned cells was determined by MTT and Transwell Matrigel invasion assays (x200, magnification), respectively. ${ }^{*} \mathrm{P}<0.05$ vs. miR-NC. ${ }^{* *} \mathrm{P}<0.05$ vs. miR-889 mimics+pcDNA3.1. TAB1, TGF- $\beta$-activated kinase 1-binding protein 1; NSCLC, non-small cell lung cancer.

A

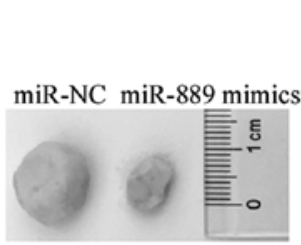

$\mathrm{B}_{2000}$

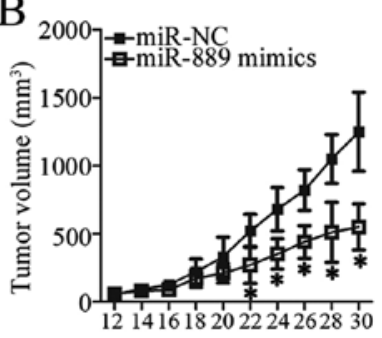

E

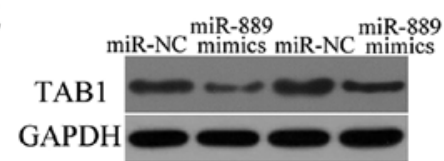

C

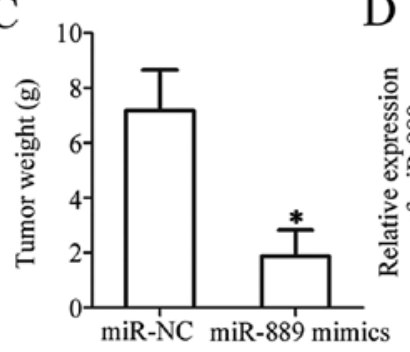

$\mathrm{D}$

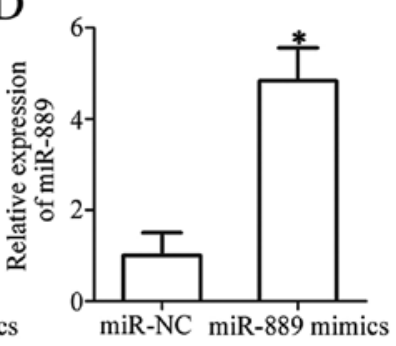

Figure 6. miR-889 upregulation decreases tumor growth in vivo. (A) Representative images of the xenografts derived from H460 cells transfected with miR-889 mimics or miR-NC. (B) The tumor volume of xenografts formed was detected every 2 days and calculated with the following formula: Volume $\left(\mathrm{mm}^{3}\right)=$ width $^{2}\left(\mathrm{~mm}^{2}\right) \times$ length $(\mathrm{mm}) / 2$. ${ }^{*} \mathrm{P}<0.05$ vs. miR-NC. (C) The xenografts were excised and weighed at the end of the experiment. ${ }^{*} \mathrm{P}<0.05 \mathrm{vs}$. miR-NC. (D) miR-889 expression in the xenografts was determined by RT-qPCR. ${ }^{*} \mathrm{P}<0.05$ vs. miR-NC. (E) The expression level of TAB1 protein in xenografts was determined by western blot analysis.

study, for the first time, miR-889 expression was detected and its clinical value in NSCLC was determined. Furthermore, the functional role and underlying mechanism of miR- 889 in
NSCLC was investigated. The results from this study indicated that miR-889 may serve as a tumor-suppressive miRNA in NSCLC in vitro and in vivo by directly targeting TAB1. 
miR-889 has been revealed to be involved in tumor development and progression of esophageal squamous cell carcinoma (17) and hepatocellular carcinoma (18). miR-889 was revealed to be expressed at high levels in esophageal squamous cell carcinoma tissues and cell lines. miR-889 restoration promoted the proliferation of esophageal squamous cell carcinoma cells in vitro and in vivo (17). In hepatocellular carcinoma, expression of miR-889 was negatively regulated by histone deacetylase inhibitors. Functionally, ectopic miR-889 expression reduced the susceptibility of hepatocellular carcinoma cells to natural killer (NK) lysis. However, the expression pattern and functions of miR-889 in NSCLC are still unknown. In the study, miR-889 expression was revealed to be decreased in NSCLC tissues and cell lines. Low miR-889 expression was notably correlated with the TNM stage and distant metastasis of NSCLC patients. Overexpression of miR-889 suppressed NSCLC cell proliferation and invasion in vitro as well as inhibited tumor growth in vivo. These findings indicated that miR-889 may be an effective therapeutic target for patients with the aforementioned human cancer types.

Two genes, DOC-2/DAB2 interactive protein (17) and major histocompatibility complex class I chain-related gene B (18), have been previously identified as the direct target genes of miR- 889 . In the present study, TAB1 was demonstrated to be a direct and functional downstream target of miR-889 in NSCLC cells. Increasing evidence has revealed that TAB1 is upregulated in a variety of human cancers, including colorectal (24), breast (25) and ovarian cancer (26). Upregulated TAB1 expression may be closely associated with tumorigenesis and tumor development through regulation of various aggressive behaviors $(24,26,27)$. In NSCLC, TAB1 was upregulated in tumor tissues, and upregulation of TAB1 was correlated with clinical stage and lymph node metastasis (20). Patients with NSCLC and higher TAB1 expression exhibited a significantly lower 5-year survival rate than those patients with lower TAB1 expression (20). However, the functional roles of TAB1 in NSCLC remain largely to be elucidated. Herein, we revealed that TAB1 silencing inhibited the proliferative and invasive abilities of NSCLC cells in vitro. miR-889 was capable of directly targeting TAB1 and inhibiting the malignant progression of NSCLC cells in vitro and in vivo. Therefore, TAB1 knockdown via miR-889 reintroduction may be a suitable therapeutic technique for the management of patients with NSCLC.

In summary, the significant downregulation of miR-889 was observed in NSCLC and it was demonstrated that miR-889 expression was associated with TNM stage and distant metastasis. It was further demonstrated that miR-889 suppressed NSCLC cell proliferation, invasion in vitro and decreased tumor growth in vivo, at least partly, by directly targeting TAB1. The present findings indicate that miR-889 may function as a tumor suppressor miRNA in NSCLC and holds promise as a potential diagnosis biomarker and therapeutic target for patients with this fatal disease.

\section{Acknowledgements}

Not applicable.

\section{Funding}

No funding was received.

\section{Availability of data and materials}

The datasets used and/or analyzed during the present study are available from the corresponding author on reasonable request.

\section{Authors' contributions}

All authors significantly contributed to the findings and methods. XW designed this research and analyzed the data. ZD and BL performed all functional experiments. All authors read and approved the manuscript and agree to be accountable for all aspects of the research in ensuring that the accuracy or integrity of any part of the work are appropriately investigated and resolved.

\section{Ethics approval and consent to participate}

The present study was approved by the Ethics Committee of Weifang People's Hospital (Weifang, China), and was performed in accordance with the Declaration of Helsinki and the guidelines of the Ethics Committee of Weifang People's Hospital. Written informed consent was also provided by all patients.

\section{Patient consent for publication}

Not applicable.

\section{Competing interests}

The authors declare that they have no competing interests.

\section{References}

1. Ferlay J, Shin HR, Bray F, Forman D, Mathers C and Parkin DM: Estimates of worldwide burden of cancer in 2008: GLOBOCAN 2008. Int J Cancer 127: 2893-2917, 2010.

2. Siegel RL, Miller KD and Jemal A: Cancer statistics, 2017. CA Cancer J Clin 67: 7-30, 2017.

3. Qin H, Wang F, Liu H, Zeng Z, Wang S, Pan X and Gao H: New advances in immunotherapy for non-small cell lung cancer. Am J Transl Res 10: 2234-2245, 2018.

4. Li Z, Song Y, Liu L, Hou N, An X, Zhan D, Li Y, Zhou L, Li P, Yu L, et al: miR-199a impairs autophagy and induces cardiac hypertrophy through mTOR activation. Cell Death Differ 24: 1205-1213, 2015.

5. Mao M, Wu Z and Chen J: MicroRNA-187-5p suppresses cancer cell progression in non-small cell lung cancer (NSCLC) through down-regulation of CYP1B1. Biochem Biophys Res Commun 478: 649-655, 2016.

6. Verdecchia A, Francisci S, Brenner H, Gatta G, Micheli A, Mangone L and Kunkler I; Eurocare-4 Working Group: Recent cancer survival in Europe: A 2000-02 period analysis of EUROCARE-4 data. Lancet Oncol 8: 784-796, 2007.

7. Li Z and Rana TM: Therapeutic targeting of microRNAs: Current status and future challenges. Nat Rev Drug Discov 13: 622-638, 2014.

8. Behm-Ansmant I, Rehwinkel J and Izaurralde E: MicroRNAs silence gene expression by repressing protein expression and/or by promoting mRNA decay. Cold Spring Harb Symp Quant Biol 71: 523-530, 2006.

9. Ameres SL and Zamore PD: Diversifying microRNA sequence and function. Nat Rev Mol Cell Biol 14: 475-488, 2013.

10. Florczuk M, Szpechcinski A and Chorostowska-Wynimko J: miRNAs as biomarkers and therapeutic targets in non-small cell lung cancer: Current perspectives. Target Oncol 12: 179-200, 2017.

11. Xu BB, Gu ZF, Ma M,Wang JY and Wang HN: MicroRNA-590-5p suppresses the proliferation and invasion of non-small cell lung cancer by regulating GAB1. Eur Rev Med Pharmacol Sci 22: 5954-5963, 2018. 
12. Boldrini L, Giordano M, Lucchi M, Melfi F and Fontanini G: Expression profiling and microRNA regulation of the LKB1 pathway in young and aged lung adenocarcinoma patients. Biomed Rep 9: 198-205, 2018.

13. Pan Q, Sun L, Zheng D, Li N, Shi H, Song J, Shao G and Xu G: MicroRNA-9 enhanced cisplatin sensitivity in nonsmall cell lung cancer cells by regulating eukaryotic translation initiation factor 5A2. Biomed Res Int 2018: 1769040, 2018.

14. Qi YJ, Zha WJ and Zhang W: MicroRNA-217 alleviates development of non-small cell lung cancer by inhibiting AKT3 via PI3K pathway. Eur Rev Med Pharmacol Sci 22: 5972-5979, 2018.

15. Lu J, Zhan Y, Feng J, Luo J and Fan S: MicroRNAs associated with therapy of non-small cell lung cancer. Int J Biol Sci 14 390-397, 2018

16. Fadejeva I, Olschewski H and Hrzenjak A: MicroRNAs as regulators of cisplatin-resistance in non-small cell lung carcinomas. Oncotarget 8: 115754-115773, 2017.

17. Xu Y, He J, Wang Y, Zhu X, Pan Q, Xie Q and Sun F: miR-889 promotes proliferation of esophageal squamous cell carcinomas through DAB2IP. FEBS Lett 589: 1127-1135, 2015.

18. Xie H, Zhang Q, Zhou H, Zhou J, Zhang J, Jiang Y, Wang J, Meng X, Zeng L and Jiang X: microRNA-889 is downregulated by histone deacetylase inhibitors and confers resistance to natural killer cytotoxicity in hepatocellular carcinoma cells. Cytotechnology 70: 513-521, 2018.

19. Livak KJ and Schmittgen TD: Analysis of relative gene expression data using real-time quantitative PCR and the 2(-Delta Delta C(T)) method. Methods 25: 402-408, 2001.
20. Zhu J, Li Q, He JT and Liu GY: Expression of TAK1/TAB1 expression in non-small cell lung carcinoma and adjacent normal tissues and their clinical significance. Int J Clin Exp Pathol 8 15801-15807, 2015

21. Zhou Q, Huang SX, Zhang F, Li SJ, Liu C, Xi YY, Wang L, Wang X, He QQ, Sun CC and Li DJ: MicroRNAs: A novel potential biomarker for diagnosis and therapy in patients with non-small cell lung cancer. Cell Prolif 50, 2017.

22. Han Y and Li H: miRNAs as biomarkers and for the early detection of non-small cell lung cancer (NSCLC). J Thorac Dis 10: 3119-3131, 2018

23. Li L, Sun Y, Feng M, Wang L and Liu J: Clinical significance of blood-based miRNAs as biomarkers of non-small cell lung cancer. Oncol Lett 15: 8915-8925, 2018.

24. Gong H, Fang L, Li Y, Du J, Zhou B, Wang X, Zhou H, Gao L, Wang $\mathrm{K}$ and Zhang J: miR873 inhibits colorectal cancer cell proliferation by targeting TRAF5 and TAB1. Oncol Rep 39: 1090-1098, 2018.

25. Neil JR and Schiemann WP: Altered TAB1:I kappaB kinase interaction promotes transforming growth factor beta-mediated nuclear factor-kappaB activation during breast cancer progression. Cancer Res 68: 1462-1470, 2008.

26. Shuang T, Wang M, Zhou Y, Shi C and Wang D: NF-kappaB1, c-Rel, and ELK1 inhibit miR-134 expression leading to TAB1 upregulation in paclitaxel-resistant human ovarian cancer. Oncotarget 8: 24853-24868, 2017.

27. Isono T, Kim CJ, Ando Y, Sakurai H, Okada Y and Inoue H: Suppression of cell invasiveness by periostin via TAB1/TAK1. Int J Oncol 35: 425-432, 2009. 